\title{
Avaliação do uso da Escala Modificada de Borg na crise asmática
}

\author{
Tatiane G. de Liz Cavallazzi ${ }^{1}$ \\ Rodrigo S. Cavallazzi \\ Tatiana de Medeiros Colletti Cavalcante ${ }^{3}$ \\ Ana Rita de Cássia Bettencourt ${ }^{4}$ \\ Solange Diccini ${ }^{5}$
}

Cavallazzi TGL, Cavallazzi RS, Cavalcante TMC, Bettencourt ARC, Diccini S. Avaliação do uso da Escala Modificada de Borg na crise asmática Acta Paul Enferm 2005; 18(1):39-45.

\begin{abstract}
RESUMO: A dispnéia é a maior causa de incapacidade e redução da qualidade de vida para pacientes com doenças respiratórias como é o caso da asma. Os objetivos deste estudo foram verificar o grau de dispnéia dos pacientes em crise asmática pela Escala Modificada de Borg e a correlação da melhora do grau de dispnéia com a melhora da função pulmonar verificada pelo Pico de Fluxo Expiratório (PFE). Foram incluídos 40 pacientes asmáticos em crise aguda, atendidos no serviço de Pronto Atendimento em Pneumologia do Hospital São Paulo, no período de setembro à dezembro de 2003. Antes e após o tratamento, os seguintes dados foram coletados: freqüência cardíaca $(F C)$, freqüência respiratória (FR), PFE e saturação periférica de oxigênio (SpO ). O paciente era questionado sobre sua percepção da dispnéia pela Escala Modificada de Borg. ${ }^{2}$ No pré-tratamento ocorreu uma tendência dos valores altos da escala de Borg estarem relacionados aos valores baixos do PFE, invertendo esta relação no pós-tratamento. Esta escala é rápida, barata e de fácil aplicabilidade na avaliação de pacientes asmáticos. Pode ser utilizada por profissionais de saúde para a avaliação inicial dos pacientes em crise, bem como sua resposta ao tratamento.
\end{abstract}

Descritores: Enfermagem; Doença pulmonar; Dispnéia. Asma

- Artigo recebido em 30/07/04 e aprovado em 27/01/05

\section{INTRODUÇÃO}

Em pacientes com doenças respiratórias, o sintoma de dispnéia é um dos mais comuns e pode demonstrar uma complicação significativa. A sensação de dispnéia é uma experiência sensorial que é percebida, interpretada e medida pelo indivíduo ${ }^{(1)}$. A dificuldade ou desconforto respiratório é provavelmente o fator isolado mais importante, na limitação da capacidade do indivíduo nas funções básicas do dia-a-dia, fazendo com que este procure logo o serviço de saúde ${ }^{(2)}$. Os pacientes expressam a sensação de dispnéia de diferentes formas como "falta de ar", "angústia no peito" ou em termos de incapacidade funcional como: "não posso mais limpar minha casa”(3).
Asma é uma doença inflamatória crônica das vias aéreas, potencialmente fatal, caracterizada por hiper-reatividade brônquica a estímulos variados. Afeta cerca de $10 \%$ da população, sendo considerada um sério problema de saúde pública ${ }^{(4)}$. No Brasil, em 1996, a asma foi a quarta causa de internação nos hospitais do Sistema Único de Saúde e é responsável por uma média de 2000

1 Enfermeira Especialista em Pneumologia modalidade residência pela Universidade Federal de São Paulo - UNIFESP. Médico Especialista em Pneumologia pela UNIFESP

3 Enfermeira Especialista em Cardiologia modalidade residência pela UNIFESP. Enfermeira técnico-administrativa do Departamen to de Enfermagem da UNIFESP

4 Doutor em Ciências; Professor Adjunto da Disciplina de Fundamentos de Enfermagem e Enfermagem Médico Cirúrgica do Departamento de Enfermagem da UNIFESP. E-mail anarita@denf.epm.br

5 Doutor em Ciências; Professor Adjunto da Disciplina de Fundamentos de Enfermagem e Enfermagem Médico Cirúrgica do Departamento de Enfermagem da UNIFESP 
óbitos/ano, aproximadamente $70 \%$ destes, durante hospitalizações dos pacientes $^{(5)}$. A dispnéia na asma resulta da dissociação entre a atividade do centro respiratório motor e dos estímulos aferentes dos receptores das vias aéreas. $\mathrm{O}$ desequilíbrio entre a força gerada pelos músculos respiratórios e as alterações resultantes no comprimento muscular e nos volumes pulmonares, constitui uma desvantagem mecânica adicional durante a inspiração. Estímulos aferentes de receptores vagais, mecanorreceptores localizados na parede torácica e quimiorreceptores são responsáveis pela retroalimentação de informações para o tronco cerebral. O trabalho respiratório e as pressões intrabrônquicas e transtorácicas alteradas parecem contribuir para o desconforto da dispnéia ${ }^{(4)}$.

A avaliação do paciente com dispnéia, que chega ao serviço de emergência ou ao serviço de pronto-atendimento, usualmente inclui freqüência respiratória, oximetria de pulso (para verificar a saturação periférica de oxigênio, $\mathrm{SpO}_{2}$ ), cor da pele, ausculta respiratória, habilidade de falar e o que desencadeou o episódio. Alguns serviços fazem a medida de pico de fluxo expiratório (PFE) como parte da avaliação em pacientes asmáticos, mas esta é uma prática pouco comum no Brasil. De qualquer modo, os pacientes não são rotineiramente questionados sobre a intensidade ou grau de sua dispnéia. Em uma doença crônica como a asma, a opinião do paciente em relação à sua percepção de melhora ou piora exerce uma importante influência na determinação da necessidade de medicamentos e uso de serviços de saúde ${ }^{(6)}$. A avaliação inadequada por médicos e pacientes pode retardar a identificação da obstrução brônquica, concorrendo para tratamento inadequado e morte inesperada de pacientes ${ }^{(7)}$.

Alguns pacientes são incapazes de perceber a intensidade da obstrução brônquica, subestimando a gra- vidade da doença. Esses pacientes apresentam poucos sintomas a despeito da intensa obstrução.

A literatura mostra que vários instrumentos foram propostos para avaliação da intensidade da dispnéia, incluindo métodos de entrevista, questionários auto-aplicados e escalas numéricas e visuais ${ }^{(8)}$. A Escala Modificada de Borg, pode ser utilizada para quantificar a dispnéia e a sua aplicação é feita de forma direta, no momento em que o paciente está apresentando a dispnéia ${ }^{(8)}$.

Assim, este estudo tem por objetivos verificar o grau de dispnéia dos pacientes com crise de asma utilizando a Escala Modificada de Borg, no pré e pós-tratamento e analisar a correlação entre a melhora do grau de dispnéia pela Escala Modificada de Borg com a melhora da função pulmonar, no pré e pós-tratamento.

\section{MÉTODO}

Estudo do tipo transversal realizado no serviço de pronto atendimento em Pneumologia do Hospital São Paulo, no período de setembro a dezembro de 2003.

A amostra foi constituída por pacientes com diagnóstico de crise asmáti$\mathrm{Ca}^{(5)}$, de ambos os sexos, com idade superior à 16 anos. Foram excluídos pacientes portadores de outras patologias capazes de produzir dispnéia (cardiopatia, doenças musculares e outras doenças pulmonares).

Este estudo foi aprovado pelo Comitê de Ética em Pesquisa da UNIFESP e os pacientes assinaram o termo de consentimento livre e esclarecido.

$\mathrm{O}$ atendimento inicial do paciente era feito pela equipe médica do Serviço de Pronto Atendimento de Pneumologia, o qual realizava o diagnóstico de crise asmática e prescrevia o tratamento, seguindo o algoritmo do Consenso Brasileiro no Manejo da Asma ${ }^{(5)}$. Os medicamentos prescritos foram $\mathrm{B}_{2}$-agonistas e o Brometo de Ipratrópio por nebulização contínua com fluxo de oxigênio de $6 \mathrm{l} / \mathrm{min}$, ou através de aerossol dosimetrado com espaçador de grande volume $(650 \mathrm{ml})$.

Na sala de inalação, antes da administração da terapêutica (prétratamento) e na alta do paciente (pós-tratamento), um dos pesquisadores coletou os seguintes dados: $\mathrm{SpO}_{2}$, freqüência cardíaca (FC), freqüência respiratória (FR) e PFE. O paciente era questionado sobre sua percepção de dispnéia, pela aplicação da Escala Modificada de Borg ${ }^{(8)}$. Esta é uma escala vertical quantificada de 0 a 10 , onde 0 representa nenhum sintoma e 10 representa sintoma máximo (Figura 1). Os pacientes eram orientados para escolher uma única pontuação que refletisse o seu grau de dispnéia, no pré e póstratamento.

\begin{tabular}{|cc|}
\hline 0 & Nenhuma \\
0,5 & Muito, muito leve \\
1 & Muito leve \\
2 & Leve \\
3 & Moderada \\
4 & Pouco intensa \\
5 & Intensa \\
6 & \\
7 & Muito intensa \\
8 & \\
9 & Muito, muito intensa \\
10 & Máxima \\
\hline
\end{tabular}

Figura 1 - Escala Modificada de Borg

Na análise estatística, as variações encontradas nos parâmetros FC, FR e $\mathrm{SpO}_{2}$, foram avaliadas pelo teste $\mathrm{t}$ de Student. Foi utilizado o coeficiente de correlação por postos de Spearman para avaliar a associação entre as variações nos parâmetros PFE e a Escala Modificada de Borg. Adotou-se o nível de significância de $0,05(\mathrm{a}=5 \%)$ e níveis descritivos (p) inferiores a esse valor foram considerados significante e representados por *.

\section{RESULTADOS}

Foram estudados 40 pacientes com diagnóstico de crise de asma. 
No quadro 1 podemos observar o grau de dispnéia pelos esco- res da Escala Modificada de de asma pré e pós-tratamento.

Borg dos pacientes em crise

Quadro 1 - Grau de dispnéia pelos escores da Escala Modificada de Borg dos pacientes em crise de asma pré e pós tratamento. (São Paulo, 2003)

\begin{tabular}{|c|c|c|}
\hline Paciente & $\begin{array}{c}\text { Escore da Escala Modificada de } \\
\text { Borg pré-tratamento }\end{array}$ & $\begin{array}{c}\text { Escore da Escala Modificada de } \\
\text { Borg pós-tratamento }\end{array}$ \\
\hline 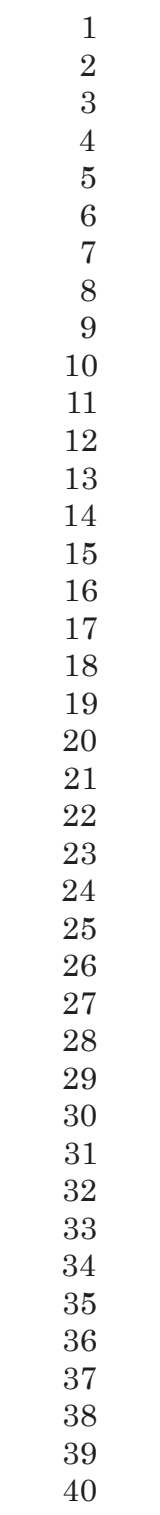 & $\begin{array}{r}5 \\
5 \\
10 \\
8 \\
5 \\
5 \\
9 \\
6 \\
10 \\
8 \\
5 \\
5 \\
4 \\
10 \\
0 \\
10 \\
4 \\
10 \\
8 \\
3 \\
5 \\
6 \\
6 \\
5 \\
5 \\
5 \\
5 \\
7 \\
6 \\
10 \\
4 \\
4 \\
10 \\
3 \\
5 \\
3 \\
10 \\
10 \\
8 \\
5 \\
5 \\
5 \\
5 \\
5 \\
5 \\
5 \\
5 \\
5 \\
5 \\
5 \\
5 \\
5\end{array}$ & $\begin{array}{l}2 \\
3 \\
3 \\
5 \\
2 \\
1 \\
4 \\
5 \\
0 \\
6 \\
2 \\
2 \\
1 \\
8 \\
0 \\
5 \\
2 \\
6 \\
4 \\
1 \\
2 \\
0 \\
3 \\
2 \\
2 \\
3 \\
2 \\
4 \\
3 \\
3 \\
2 \\
2 \\
2 \\
1 \\
1 \\
2 \\
5 \\
0 \\
0 \\
5\end{array}$ \\
\hline
\end{tabular}

No Quadro 2 podemos observar a média das variáveis freqüência cardíaca (FC), freqüência respiratória (FR) e saturação periférica de oxi- gênio $\left(\mathrm{SpO}_{2}\right)$ dos pacientes pré e póstratamento com broncodilatadores. 
Quadro 2 - Comparação da média $( \pm d p)$ da FC, FR, SpO e PFE pré e pós-tratamento em pacientes em crise de asma. São Paulo, 2003

\begin{tabular}{cccc}
\hline Variáveis & $\begin{array}{c}\text { Média }( \pm \mathbf{d p}) \\
\text { Pré-tratamento }\end{array}$ & $\begin{array}{c}\text { Média ( } \pm \text { dp) } \\
\text { Pós-tratamento }\end{array}$ & $\mathbf{p}$ \\
\hline FC (bpm) & $85,9(16,4)$ & $89,7(17,2)$ & $\mathrm{p}=0,085$ \\
FR (rpm) & $22,5(4,7)$ & $19,6(4,2)$ & $\mathrm{p}=0,002^{*}$ \\
SpO & $95,5(1,9)$ & $96,3(1,6)$ & $\mathrm{p}=0,002^{*}$ \\
$\mathrm{PFE} \mathrm{(1/min)}$ & $202(71,1)$ & $292(78,1)$ & $\mathrm{p}<0,001^{*}$ \\
\hline
\end{tabular}

A média de freqüência cardíaca dos pacientes era de 85,9 bpm (dp = 16,4 bpm) na avaliação pré e aumentou para 89,7 bpm $(\mathrm{dp}=17,2 \mathrm{bpm})$ no pós tratamento. Apesar do aumento médio de 3,8 bpm, essa variação não se mostrou estatisticamente significante $(p=0,085)$.

A média de freqüência respiratória dos pacientes era de 22,5 rpm (dp = 4,7 rpm) na avaliação pré e dimi-

Test $t$ de Student

nuiu para 19,6 rpm (dp = 4,2 rpm) no pós-tratamento. Foi encontrada uma redução média de 2,9 rpm, sendo que essa variação se mostrou estatisticamente significante ( $\mathrm{p}=0,002)$.

A média de $\mathrm{SpO} 2$ dos pacientes era de 95,5 \% (dp = 1,9 \%) na avaliação pré e aumentou para 96,3 \% (dp $=1,6 \%)$ no pós tratamento. Foi encontrado um aumento médio de $0,78 \%$, sendo essa variação estatisticamente significante $(\mathrm{p}=0,002)$.
A média do PFE dos pacientes era de 202 l/min (dp = 71,1) na avaliação pré e aumentou para $292 \mathrm{l} / \mathrm{min}$ $(\mathrm{dp}=78,1)$ no pós-tratamento. Foi encontrado um aumento médio de $90 \mathrm{l} / \mathrm{min}$, sendo essa variação estatisticamente significante $(\mathrm{p}<0,001)$.

Outro ponto estudado foi a correlação entre PFE e os escores da Escala Modificada de Borg na avaliação pré-tratamento broncodilatador evidenciado na Figura 2.

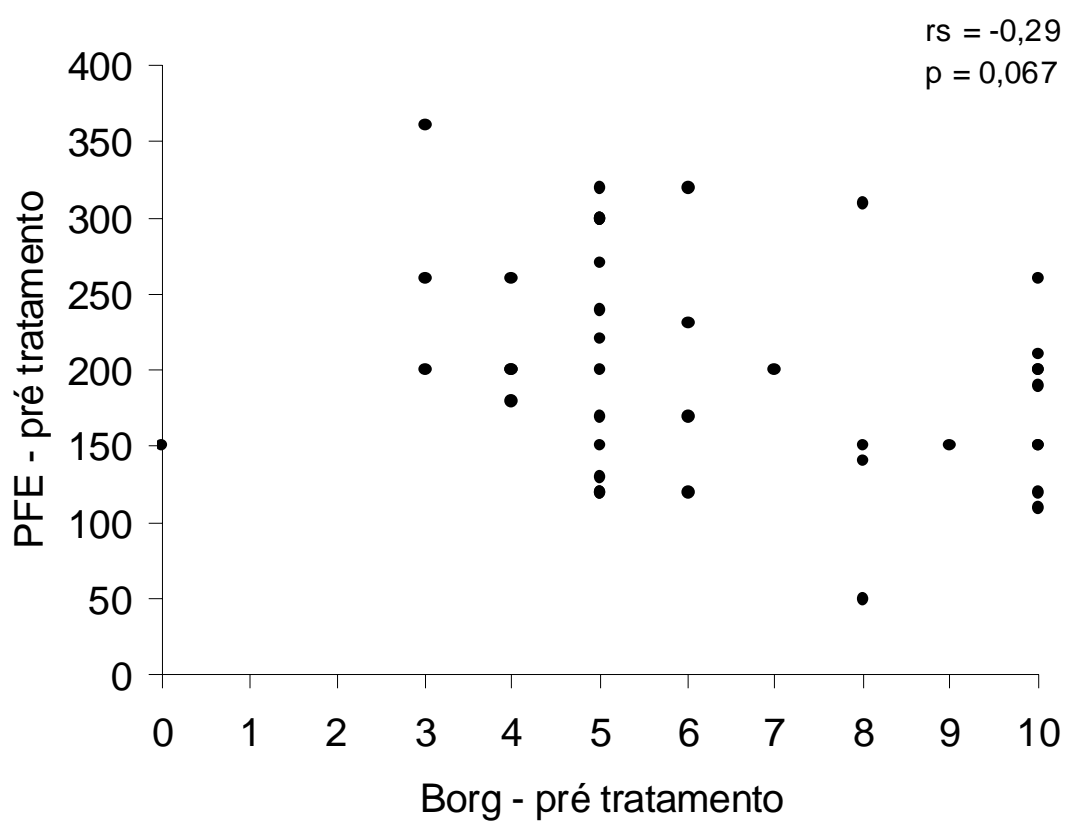

Figura 2 - Correlação entre PFE e Escala Modificada de Borg na avaliação pré-tratamento

Na avaliação pré-tratamento foi encontrada correlação negativa não significante ( $r s=-0,29 \mathrm{p}=0,067$ ). $\mathrm{O}$ resultado mostra uma tendência de os valores altos da Escala de Borg estarem relacionados aos valores baixos de PFE, porém não evidenciada estatisticamente.
A Figura 3 demonstra a correlação entre PFE e os valores da Escala Modificada de Borg na avaliação pós-tratamento broncodilatador. 


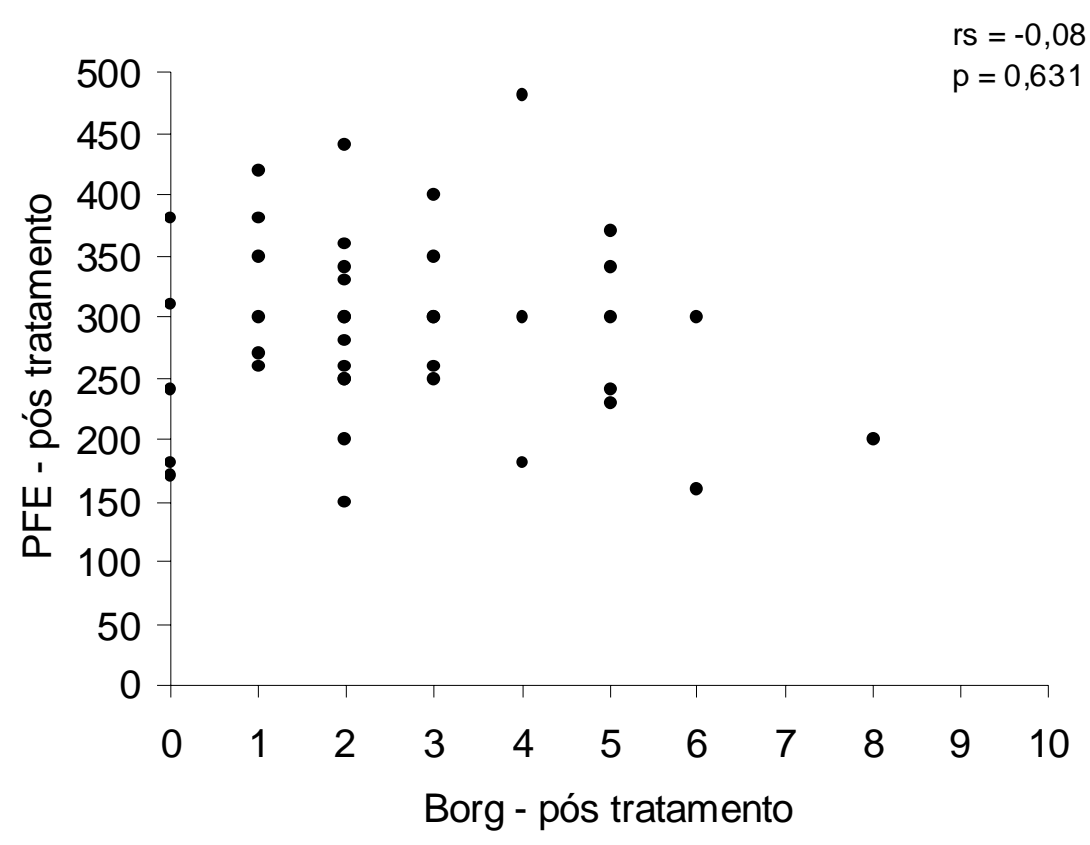

Figura 3 - Correlação entre PFE e Escala Modificada de Borg na avaliação pós-tratamento

Na avaliação pós-tratamento foi encontrada correlação negativa não significante ( $r s=-0,08 \mathrm{p}=0,631)$.

\section{DISCUSSÃO}

As crises de asma habitualmente evoluem com progressiva obstrução brônquica em intervalos que variam de algumas horas até muitos dias. Em alguns pacientes os episódios de asma caracterizam-se por início abrupto e inesperado, podendo ocasionar a morte em alguns minutos ${ }^{(7)}$.

Neste estudo, observou-se que após a instituição da terapêutica broncodilatadora, os valores da Escala Modificada de Borg, sofreram decréscimo importante em relação ao pré-tratamento, na maioria dos casos. Isto reflete, mesmo que indiretamente, a melhora da dispnéia após o tratamento, dado este já demonstrado em algumas pesquisas ${ }^{(9-11)}$.

O Consenso Brasileiro no Manejo da Asma, em 2002, preconiza a utilização de parâmetros objetivos como a $\mathrm{SpO}_{2}$ e o PFE, além da avaliação clínica habitual no manejo da crise de asma. De acordo com determinadas situações, preconiza também a realização de gasometria arterial, radiografia do tórax, hemograma e eletrólitos ${ }^{(5)}$. Consideramos necessárias e importantes a utilização de ferramentas adicionais na avaliação de pacientes com crise de asma e sua resposta ao tratamento.

Observou-se neste estudo, uma relação estatisticamente significante entre a freqüência respiratória, a saturação de oxigênio e o PFE, no pré e pós-tratamento. Em todos estes parâmetros respiratórios descritos, houve uma melhora significativa nos valores pré e pós-tratamento, demonstrando assim a eficácia do tratamento proposto. Segundo estudos, além da modificação do ambiente e do comportamento do asmático, é essencial que se dê atenção ao controle ou avaliação da sintomatologia, principalmente a dispnéia, a tosse e a sensação de opressão torácica ${ }^{(12)}$. Para isso o esquema medicamentoso deve conter broncodilatador. O uso da medida de pico de fluxo expiratório (PFE) como indicador de calibre brônquico e como preditor das agudizações é recomendado na literatura especializada ${ }^{(12)}$.

Parâmetros objetivos de função pulmonar são importantes para avaliação da intensidade da obstrução brônquica em asmáticos. No presente estudo evidenciamos que no pré-tratamento valores altos da Escala Modificada de Borg estão associados a valores baixos de PFE, correlação esta invertida no pós-tratamento, onde valores altos de PFE estão associados a baixos valores da Escala Modificada de Borg. Diante desta realidade, podemos observar a melhora tanto da função pulmonar quanto da dispnéia, apesar da relação PFE e Escala Modificada de Borg no pré e pós-tratamento não apresentar associação significante. Estes dados foram semelhantes aos encontrados em outros estudos ${ }^{(7,9,11)}$.

A Escala Modificada de Borg nos pareceu um instrumento adicional rápido, barato e de fácil aplica- 
bilidade na avaliação de pacientes com crise de asma. Pode ser utilizada por profissionais de saúde encarregados de realizar a avaliação inicial de pacientes com crise de asma, assim como a resposta ao tratamento.

A má percepção da dispnéia por pacientes e profissionais de saúde, pode atrasar a identificação da gravidade da obstrução brônquica, retardar o tratamento adequado e contribuir para mortes inesperadas por asma ${ }^{(7)}$. Alguns pacientes são incapazes de perceber a intensidade da obstrução brônquica, subestimando a gravidade da doença. Uma pobre percepção da asma pode levar ao atraso no início do tratamento apropriado, o que é provavelmente um dos fatores principais que contribuem para a morte na asma ${ }^{(9)}$. Portanto, mensurações objetivas da limitação ao fluxo aéreo são cruciais para a avaliação de pacientes com asma moderada a grave, em serviços de emergência ${ }^{(7)}$.

A Escala Modificada de Borg tem sido utilizada principalmente para avaliar a percepção de dispnéia pelos indivíduos em situação de exercício físico, embora recentemente alguns trabalhos a tenham utilizado em pacientes com doenças pulmonares obstrutivas (asma ou DPOC) após indução de broncoconstrição ${ }^{(10,13-17)}$. A Escala Modificada de Borg foi bem sucedida na avaliação da dispnéia associada à broncoconstrição induzida em pacientes asmáticos ${ }^{(13)}$. Portanto, a literatura médica nos proporciona uma base fisiológica para a realização deste estudo.

Estudos realizados demonstraram que o escore de percepção da dispnéia é relacionado a marcadores inflamatórios na expectoração brônquica, os quais sugerem que a percepção da dispnéia assim como os marcadores inflamatórios de vias aéreas podem ser considerados para avaliação da gravidade clínica da $\operatorname{asma}^{\left({ }^{(9)}\right.}$.
Alguns autores estudaram a percepção da dispnéia associada à obstrução ao fluxo aéreo induzida por histamina em 45 asmáticos, utilizando a Escala Modificada de Borg ${ }^{(11)}$. A dispnéia estava presente em pacientes com e sem obstrução ao fluxo aéreo no início do estudo, mas pacientes com obstrução ao fluxo aéreo obtiveram graus similares de dispnéia somente quando eram consideravelmente mais obstruídos pela indução. Estes autores sugerem que a adaptação temporal pode ser responsável pela variabilidade da dispnéia em asmáticos, logo, a adaptação temporal limitaria a utilidade da Escala Modificada de Borg na avaliação inicial de pacientes com crise de asma, uma vez que pacientes mais obstruídos podem ter grau de dispnéia similar a outros pacientes menos graves ${ }^{(11)}$. No entanto, não invalida o seu uso na avaliação da resposta ao tratamento, como mostramos neste estudo.

Uma das limitações deste estudo foi o pequeno número de pacientes (erro tipo II) e o fato de que a percepção de dispnéia é influenciada de forma direta pelas condições emocionais e psicológicas dos pacientes além dos fatores fisiopatológicos envolvidos. A ausência de significância estatística nas correlações analisadas indica a necessidade de mais estudos sobre este assunto. Entretanto os pacientes tratados tiveram uma diminuição estatisticamente significante na sua pontuação e a escala se mostrou de fácil aplicabilidade.

\section{CONCLUSÃO}

Como esperado, os resultados deste estudo mostraram que houve uma diminuição significativa da pontuação da Escala Modificada de Borg, da freqüência respiratória e o aumento significante da SpO2 e PFE após a instituição do tratamento. Chama a atenção o aumento médio de 90 l/min do PFE. Não houve corre- lação estaticamente significante entre as pontuações da Escala Modificada de Borg e as medidas do PFE, nem antes nem após o tratamento. O uso da Escala Modificada de Borg não substitui o parâmetro fisiológico PFE na avaliação de pacientes com crise de asma e seu tratamento, mas pode ser utilizada como um recurso adicional. Acreditamos assim, que mais estudos devam ser desenvolvidos sobre este assunto.

\section{REFERÊNCIAS}

1. Kendrick KR, Smith RM. Usefulness of modif 0-10 Borg Scale in assessing degree of dyspnea in patients with COPD and Asthma. J Emerg Nurs 2000; 26(3):216-22.

2. Mahler DA, Wells CK. Evaluation of clinical methods for rating dyspnea. Chest 1988; 93(3):580-83.

3. Sociedade Brasileira de Pneumologia e Tisiologia (SBPT). Manual de pneumologia: consultas rápidas. Brasília; 2002.

4. Machado AS. Dispnéia aguda e morte súbita em pacientes com má percepção da intensidade da obstrução brônquica. J Pneumol 2001; 27(6):341-44.

5. Sociedade Brasileira de Pneumologia e Tisiologia (SBPT). III Consenso Brasileiro no Manejo da Asma. J Pneumol 2002; 28(1):28.

6. Morogón EM. Percepción de mejoría en los pacientes com asma. Arch Bronconeumal 2002; 38(10):468-72.

7. Machado AS. Má percepção da limitação aos fluxos aéreos em pacientes com asma moderada a grave. J Pneumol 2001; 27(4):185-92

8. Burneto AF. Comparação entre a escala modificada de Borg e a escala de Borg modificada análago visual aplicadas em pacientes com dispnéia. Rev Bras Ciênc Mov 1989; 3(1):34-40.

9. Jang AS, Choi IS. Relationship between the perception of dyspnoea and airway inflammatory markers. Respi Med 2002; 96:150-4. 
10. Grant S. A comparasion of the reproducibility and sensitivity to change of visual analogue scales, Borg scales, and Likert scales in normal subjects during submaximal exercise. Chest 1999;116 (5):1208-17.

11. Burdon JGW. The perception of breathlessness in asthma. Am Rev Respir Dis 1982; 126:825-8.

12. Silva JRL. Asma brônquica. In: Aidé MA. Pneumologia: aspectos práticos e atuais. Rio de Janeiro: Revinter; 2001. p. 201-10.
13. Chetta A. Assesment of breathlessness perception by Borg scale in asthmatic patients: reproducibility and applicability to different stimuli. J Asthma 2003; 40(3):323-9.

14. Pfeifer KA. Reliability and validity of the Borg and OMNI rating of perceived exertion scales in adolecent girls. Med Sci Sports Exerc 2002; 34(12):2057-61.

15. Chen MJ. Criterion-related validity of the Borg ratings of perceived exertion scale in healthy individuals: a metaanalysis. J Sports Sci 2002; 20(11): 873-99.
16. Rutgers SR. Borg scores before and after challenge with adenosine 5'monophosphate and methacholine in subjects with COPD and asthma. Eur Respir J 2000; 16(3):486-90.

17. Mador MJ. Reproducibility of Borg scale measurements of dyspnea during exercise in patients with COPD. Chest 1995; 107(6):1590-7.
Cavallazzi TGL, Cavallazzi RS, Cavalcante TMC, Bettencourt ARC, Diccini S. [Evaluation of the use of the Modified Scale of Borg in the asthmatic crisis.] Acta Paul Enferm 2005; 18(1):39-45.

ABSTRACT: The dyspnea is the biggest cause of incapacity and reduction of the quality of life for patients with respiratory illnesses as it is the case of the asthma. Being thus, the respiratory difficulty is probably the more important isolated factor in the limitation of the capacity of the individual in the basic functions of day-by-day, making with that this looks the health services. The objective of this study was to verify the degree of dyspnea of the patients in asthmatic crisis for the scored on the Modif Borg Scale and the correlation of improvement of the degree of dyspnea for the same one with the improvement of the pulmonary function verified by Peak expiratory Flow (PEF). A total of 40 asthmatic patients in acute crisis taken care of in the service of Attendance in Pneumologia of the São Paulo Hospital, were included, in the period of september to the december of 2003. Before and after the clinical treatment, the subsequent data were colected: cardiac rate (FC), respiratory rate $(\mathrm{FR}), \mathrm{PEF}$ and peripherical oxygen saturation $\left(\mathrm{SpO}_{2}\right)$ and the patient was questionado about your perception of the dyspnea for the Modif Borg Scale. The punctuation of this scale varies of 0 the 10 (of none to the dyspnea highest). The results had pointed a trend of the high values of the Modif Scale of Borg to be related to the low values of PEF in the daily pay-treatment, inverting this relation in the post-cure. This scale in them seemed a fast, cheap additional instrument and of easy applicability in the evaluation of asthmatic patients, being able to be used by health professionals who carry through the initial evaluation of the patients in crisis as well as its reply to the treatment.

Descriptors: Nursing; Pulmonary disease; Dyspnea. Asthma
Cavallazzi TGL, Cavallazzi RS, Cavalcante TMC, Bettencourt ARC, Diccini S. [Evaluación del uso de la Escala Modificada de Borg en la crisis de asma.] Acta Paul Enferm 2005; 18(1):39-45.

RESUMEN:La disnea es probablemente, el factor aislado más importante en la limitación de la capacidad de pacientes con asma para el desempeño de las funciones básicas del dia a dia. En consecuencia da esse sintoma, esos pacientes procuran con frecuencia, los servicios de atención de salud. Considerando la necesidad de uma evaluación acurada de ese síntoma, este estudio tuvo como objetivos: evaluar el grado de dísnea de pacientes en crisis de asma, utilizando la escala modificada de Borg y verificar la correlación entre la majoria del grado de dísnea y la mejoria de la función pulmonar, verificada por el Pico de Flujo Expiratorio (PFE) y la saturación de oxígeno $\left(\mathrm{SpO}_{2}\right)$. Participaron del estudio 40 pacientes en crisis aguda de asma, atendidos de septiembre a diciembre de 2003 en el servicio de Pronto Atendimento em Pneumologia del Hospital São Paulo, por medio de uma entrevista semi- estructurada que evalua a percepcion de la dísnea por parte del paciente, los datos relativos al grado da dísnea, que en la escala de Borg varía de 0 a 10 (da ausencia del síntoma al grado máximo del mismo) e la evaluación del PFE y del $\mathrm{SpO}_{2}$ fue realizada antes y depués de haberse instituido el tratamiento clínico. Los resultados apuntaron uma tendencia a que valores altos de la escala de Borg estan relacionados a valores bajos de PFE antes del tratamiento y que esta relación se inverte después del tratamiento. Nostra opinión es que esta escala constituye un instrumento adicional rápido, barato y de fácil aplicación para la evaluación inicial da essos pacientes en crisis, bien coma para la respuosta al tratamiento.

Descriptores: Enfermería; Enfermedad pulmonar; Dísnea. Asma 